\title{
Tumor-derived microRNAs induce myeloid suppressor cells and predict immunotherapy resistance in melanoma
}

\author{
Veronica Huber, ${ }^{1}$ Viviana Vallacchi, ${ }^{1}$ Viktor Fleming, ${ }^{2}$ Xiaoying Hu, ${ }^{2}$ Agata Cova, ${ }^{1}$ Matteo Dugo, ${ }^{3}$ Eriomina Shahaj, ${ }^{1}$ \\ Roberta Sulsenti, ${ }^{1}$ Elisabetta Vergani, ${ }^{1}$ Paola Filipazzi, ${ }^{1}$ Angela De Laurentiis, ${ }^{1}$ Luca Lalli, ${ }^{1}$ Lorenza Di Guardo, ${ }^{4}$ Roberto Patuzzo, ${ }^{5}$ \\ Barbara Vergani, ${ }^{6}$ Elena Casiraghi, ${ }^{7}$ Mara Cossa, ${ }^{8}$ Ambra Gualeni, ${ }^{8}$ Valentina Bollati, ${ }^{9}$ Flavio Arienti, ${ }^{10}$ Filippo De Braud, ${ }^{4}$ \\ Luigi Mariani, ${ }^{11}$ Antonello Villa, ${ }^{6}$ Peter Altevogt, ${ }^{2}$ Viktor Umansky, ${ }^{2}$ Monica Rodolfo, ${ }^{1}$ and Licia Rivoltini ${ }^{1}$ \\ ${ }^{1}$ Unit of Immunotherapy of Human Tumors, Fondazione Istituto di Ricovero e Cura a Carattere Scientifico (IRCCS) Istituto Nazionale dei Tumori di Milano, Milan, Italy. ${ }^{2}$ Skin Cancer Unit, German Cancer \\ Research Center (DKFZ), Heidelberg, and Department of Dermatology, Venereology and Allergology, University Medical Center Mannheim, Ruprecht-Karl University of Heidelberg, Heidelberg, Cermany. \\ ${ }^{3}$ Platform of Integrated Biology, ${ }^{4}$ Medical Oncology Unit 1, and ${ }^{5}$ Melanoma and Sarcoma Surgery Unit, Fondazione IRCCS Istituto Nazionale dei Tumori di Milano, Milan, Italy. ${ }^{6}$ Microscopy and Image Analysis \\ Consortium, Università degli Studi di Milano-Bicocca, Monza, Italy. ${ }^{7}$ Department of Computer Science “Ciovanni Degli Antoni," Università degli Studi di Milano, Milan, Italy. ${ }^{8}$ Molecular Pathology Unit, \\ Fondazione IRCCS Istituto Nazionale dei Tumori di Milano, Milan, Italy. ${ }^{9}$ EPICET-Epidemiology, Epigenetics and Toxicology Lab, Department of Clinical Sciences and Community Health, Università degli Studi \\ di Milano, Milan, Italy. ${ }^{10}$ Immunohematology and Transfusion Medicine, and ${ }^{11}$ Medical Statistics, Biometry and Bioinformatics, Unit of Clinical Epidemiology and Trial Organization, Fondazione IRCCS Istituto \\ Nazionale dei Tumori di Milano, Milan, Italy.
}

The accrual of myeloid-derived suppressor cells (MDSCs) represents a major obstacle to effective immunotherapy in cancer patients, but the mechanisms underlying this process in the human setting remain elusive. Here, we describe a set of microRNAs (miR-146a, miR-155, miR-125b, miR-100, let-7e, miR-125a, miR-146b, miR-99b) that are associated with MDSCs and resistance to treatment with immune checkpoint inhibitors in melanoma patients. The miRs were identified by transcriptional analyses as being responsible for the conversion of monocytes into MDSCs (CD14+HLA-DR ${ }^{\text {neg }}$ cells) mediated by melanoma extracellular vesicles (EVs) and were shown to recreate MDSC features upon transfection. In melanoma patients, these miRs were increased in circulating $\mathrm{CD} 14^{+}$monocytes, plasma, and tumor samples, where they correlated with the myeloid cell infiltrate. In plasma, their baseline levels clustered with the clinical efficacy of CTLA-4 or programmed cell death protein 1 (PD-1) blockade. Hence, MDSC-related miRs represent an indicator of MDSC activity in cancer patients and a potential blood marker of a poor immunotherapy outcome.

\section{Introduction}

Immunotherapy with immune checkpoint inhibitors (ICIs) has achieved a remarkable improvement in overall survival (OS) in different tumor histologies, albeit the majority of patients display intrinsic resistance (1). The response to programmed cell death protein 1 (PD-1) blockade requires a preexisting and flourishing immune response and involves determinants of adaptive immunity, such as a high number of tumor DNA mutations or neoantigens, the interspersed distribution of infiltrating effector $\mathrm{T}$ cells, a high level of the expression of PD-L1, or an IFN-related gene signature $(2,3)$. In contrast, resistant tumors lack signs of immunogenicity and are definable as "immune-desert" based on the absence of local T cell reactivity (4). Resistant tumors are also endowed with the innate anti-PD-1 resistance signature (IPRES) (5), which involves specific genetic programs, including wound healing,

Authorship note: VH and VV contributed equally to this work. MR and LP contributed equally to this work.

Conflict of interest: The authors have declared that no conflict of interest exists. License: Copyright 2018, American Society for Clinical Investigation.

Submitted: October 12, 2017; Accepted: September 20, 2018.

Reference information: J Clin Invest. 2018;128(12):5505-5516.

https://doi.org/10.1172/JCI98060. epithelial-mesenchymal transition (EMT), extracellular matrix remodeling, TGF- $\beta$ signaling, hypoxia, macrophage chemotaxis, and angiogenesis. Most of these mirror the transcriptional profile of myeloid-derived suppressor cells (MDSCs), a population of immature myeloid cells (IMCs) pathologically associated with cancer and known for their potent inhibitory activity on antitumor $\mathrm{T}$ cell immunity (6). In addition, MDSCs directly coordinate cancer cell plasticity, triggering the EMT phenotype $(7,8)$ and activating signaling pathways often associated with drug resistance, stroma remodeling, and angiogenesis $(9,10)$. MDSCs distribute systemically in cancer patients, accumulating in the peripheral blood, lymph nodes (LNs), and distant organs, where they facilitate cancer dissemination by forming the so-called premetastatic niche (6). A high frequency of MDSCs in the peripheral blood, mainly comprising monocytic and polymorphonuclear subsets ( 9 , $11)$, is associated with poor prognosis $(6,12)$ and represents a possible mechanism of resistance to immunotherapies (11, 13-17).

MDSC generation is believed to stem from conditioning of the $\mathrm{BM}$ by soluble tumor factors, including granulocyte-macrophage CSF (GM-CSF), macrophage CSF (M-CSF), granulocyte CSF (G-CSF), and other proinflammatory cytokines (6). A delivery of tumor factors could be facilitated by shuttling organelles (18). Extracellular vesicles (EVs), nanoparticles abundantly secreted by 
A

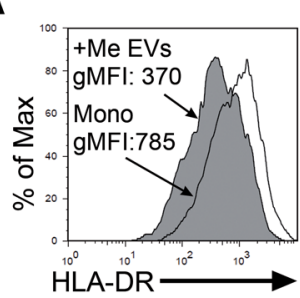

C

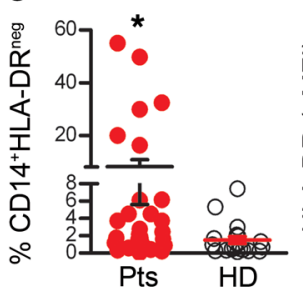

$\mathbf{F}$
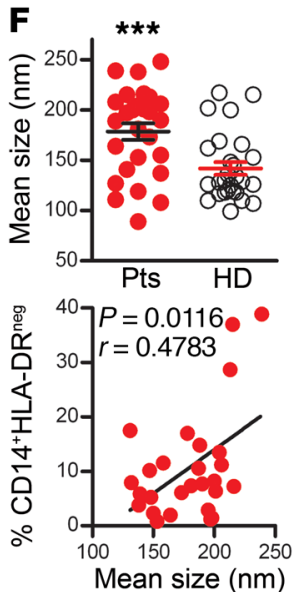
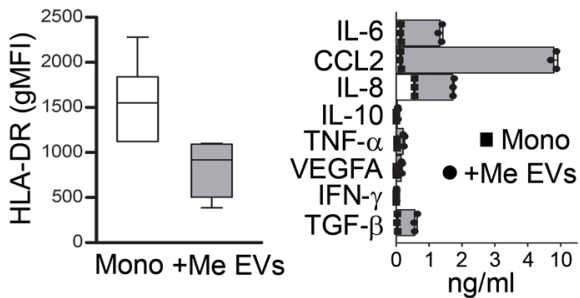

D

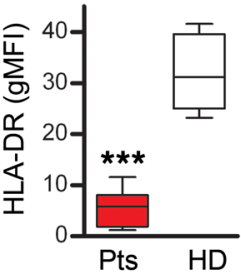

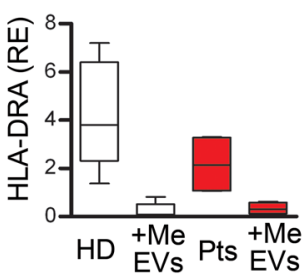

B

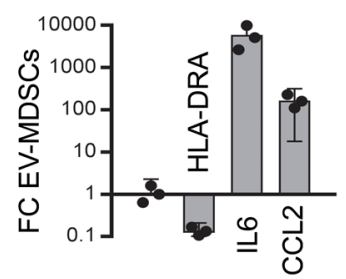

E

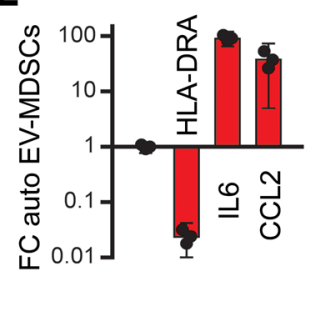

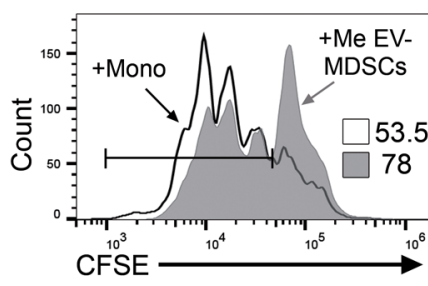

tauto

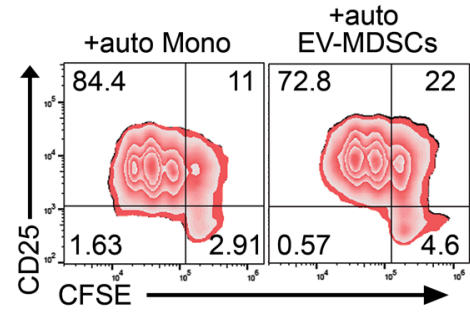

I
G

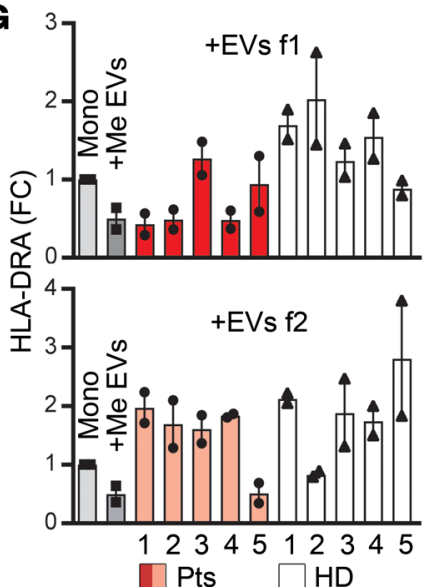

H
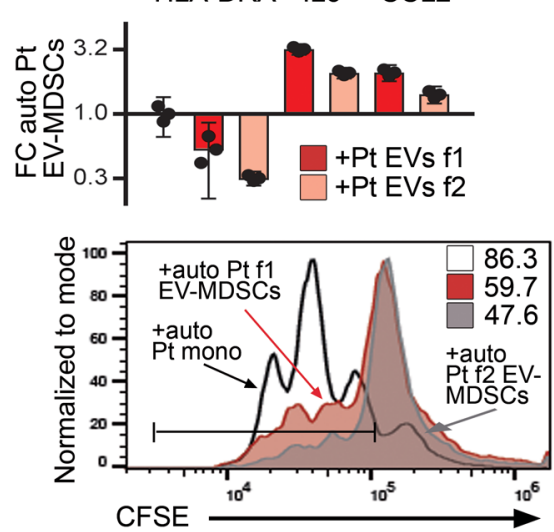

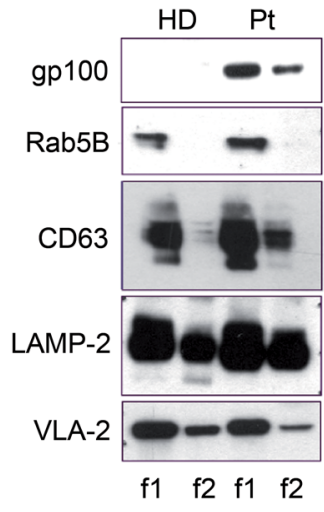

Figure 1. Conversion of myeloid cells into MDSCs by melanoma EVs. (A) HD-CD14+ cells (Mono) incubated 24 hours with melanoma EVs (Me EVs) downregulate HLA-DR (left, representative plot and summary of $n=7 \mathrm{HD}$ ), increase production of cytochemokines (middle), modulate HLA-DRA, IL6, and CCL2 gene transcription (right), and (B) suppress proliferation of activated CFSE-labeled T cells (percentage proliferation indicated). (C) CD14 ${ }^{+} H L A-D R^{\text {neg }}$ cell frequency and HLA-DR expression on gated CD14+ cells in PBMCs of melanoma patients (Pts, $n=31)$ and HD $(n=15)$ by flow cytometry. (D) HLA-DRA downregulation of HD ( $n=5)$ and patients' $(n=4)$ monocytes cultured with melanoma EVs. (E) Induction of EV-MDSCs in CD14 cells from a patient by autologous melanoma cell line EVs (left); suppressive activity on activated CD25+T cells (percentages indicated, right). (F) NTA evaluation of EV size in plasma samples of patients and HD ( $n=27 /$ group) (top); correlation of EV mean size and frequency of CD14+HLA-DR neg in gated CD14+ cells of melanoma patients (bottom). (C) EV-MDSC converting potential of $\mathrm{f} 1$ and $\mathrm{f} 2$ plasma EVs from patients and $\mathrm{HD}$ ( $n=5 /$ group) shown as HLA-DRA downregulation in monocytes from 2 different HD; control: melanoma EVs. Data are presented as mean $\pm \mathrm{SEM}$. (H) Autologous (auto) plasma EVs $\mathrm{f} 1$ and $\mathrm{f} 2$ convert melanoma patient's CD14 ${ }^{+}$cells, as shown by modulation of $H L A-D R A$, IL6, and CCL2 transcripts (top). EV-MDSCs generated with autologous plasma EVs $\mathrm{f} 1$ and $\mathrm{f} 2$ of melanoma patient inhibit T cell proliferation (percentages indicated, bottom). (I) Western blot of plasma EV fractions ( $\mathrm{f1}, \mathrm{f2})$ of $\mathrm{HD}$ and patient. gMFI, geo mean fluorescence intensity; $\mathrm{RE}$, relative expression. $\mathrm{FC}$ was by using as calibrator untreated monocytes. $P<0.001$ (A, right; E); $P<0.01$ (A, left); $P<0.05$ (H, top), paired Student's $t$ test. $P<0.05$, Mann-Whitney $U$ test $(\mathbf{D})$. ${ }^{*} P<0.05$, ${ }^{* *} P<0.001$, unpaired Student's $t$ test $(\mathbf{C}, \mathbf{F})$. Data are representative of $2(\mathbf{E}, \mathbf{H}, \mathbf{I})$ and $3(\mathbf{A}, \mathbf{B})$ experiments.

cancer cells, are involved in the intercellular transfer of proteins, lipids, and genetic material, such as RNAs and microRNAs (miRs) (19-21). Because of their ability to recirculate in body fluids, EVs represent an ideal candidate in the process of BM conditioning that leads to MDSC generation $(22,23)$. In the present work, we identified a panel of circulating miRs associated with monocytic MDSC (M-MDSC) activity in melanoma patients, representing a potential tool to assess the role of these cells in resistance to ICIs.

\section{Results}

The monocyte-MDSC transition is mediated by melanoma EVs. We and others have previously defined cancer-associated human
M-MDSCs as CD11 $\mathrm{b}^{+} \mathrm{CD} 33^{+}$myeloid cells expressing the hallmark CD14 ${ }^{+} H L A-D^{\text {neg }}$ phenotype and exerting immunosuppressive activity on $T$ cells $(9,11)$. In an attempt to mimic this phenotype in vitro, we opted to use tumor EVs to convert normal myeloid cells. EVs were purified by differential centrifugation of conditioned medium (CM) from melanoma cell lines and evaluated for their morphology and marker profiles (Supplemental Figure 1A; supplemental material available online with this article; https:// doi.org/10.1172/JCI98060DS1). Here, we report that CD14 ${ }^{+}$ monocytes, when exposed for 24 hours to melanoma EVs isolated from short-term (INT12, LM38) and long-term (Mel501, 624.38) cultured cell lines, show downregulated HLA-DR expression and 
A

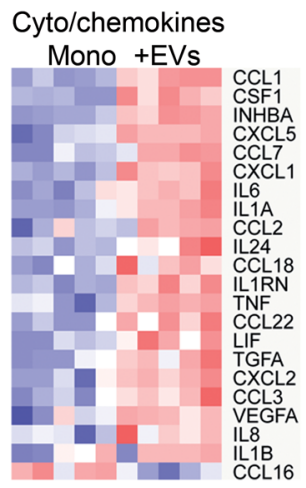

B

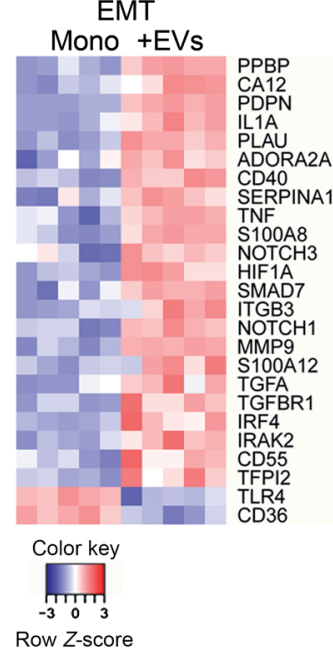

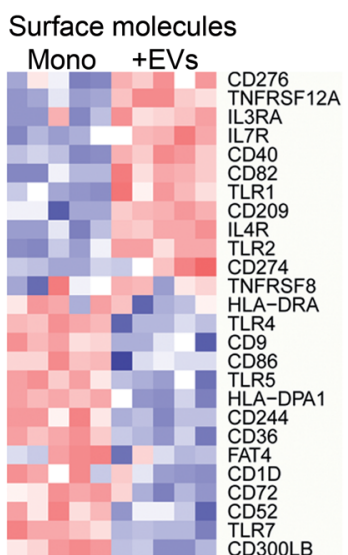

C

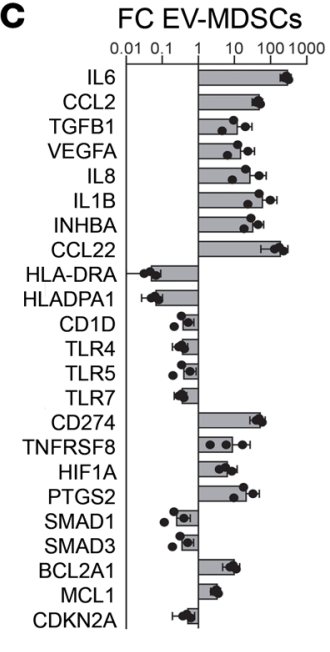

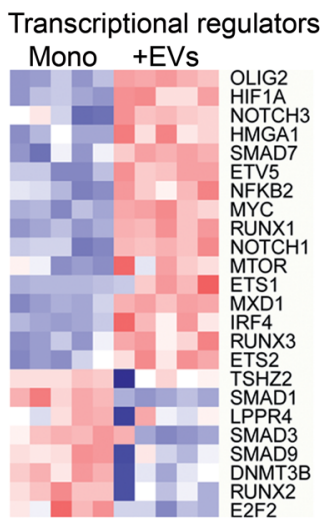

Proliferation and apoptosis

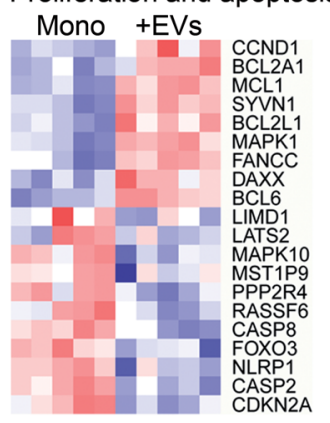

D

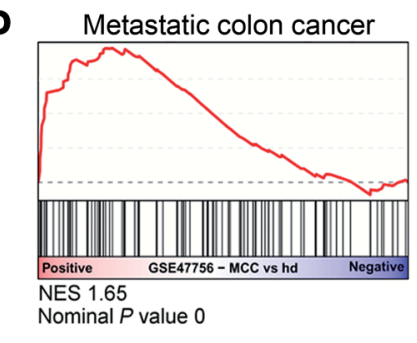

Pancreatic adenocarcinoma

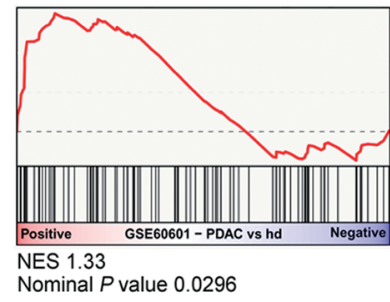

Nonmetastatic colon cancer

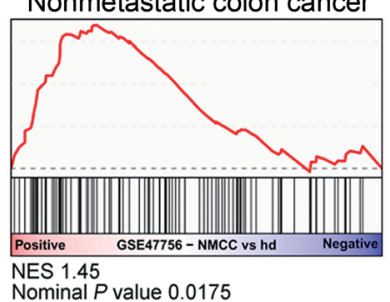

Breast cancer

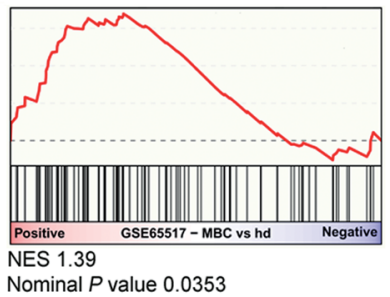

Figure 2. Transcriptional regulation of EV-MDSCs at the gene level. (A) Heatmaps of the genes regulated in EV-MDSCs (+EVs) compared with untreated monocytes (Mono, $n=5 \mathrm{HD}$ ) clustered according to the most representative functional categories. (B) EMT-related genes selected by a list of 839 genes from melanoma IPRES, EMT, and wound healing (5); from the literature-based EMT database (32); and from mesenchymal myeloid markers (33). (C) The FC of expression levels of selected genes in EV-MDSCs compared with untreated monocytes measured by qPCR. $P<0.01$, paired Student's $t$ test. A representative HD of 5 tested from part A is shown. (D) GSEA plots obtained with the top 100 upregulated genes in EV-MDSCs showing a significant enrichment in data sets comparing the transcriptomic profiles of blood monocytes from colorectal, pancreatic, and breast cancer patients to those of monocytes obtained from HD. GSE47756, colon cancer (37); GSE60601, pancreatic cancer (38); GSE65517, metastatic breast cancer (39).

increase of IL-6 and CCL2 transcription and secretion together with suppressive properties on activated T cells (Figure 1, A and B, and Supplemental Figure 1, B and C), a phenotype we have named EV-MDSCs. Monocyte conditioning strictly relies on the presence of vesicles, as EV-depleted melanoma CM loses the ability to downregulate HLA-DR expression (Supplemental Figure 1D).

CD14 ${ }^{+}$HLA-DR $^{\text {neg }}$ M-MDSCs accumulate in the peripheral blood of metastatic melanoma patients, as compared with healthy donors (HD) (Figure 1C) $(11,24)$, and show spontaneous production of immunosuppressive and protumor factors, such as IL-6, CCL2, and TGF- $\beta(10,11,25)$, detected ex vivo as RNA and proteins (Supplemental Figure 1E). Their suppressive potential is documented by the boost or decrease of $\mathrm{T}$ cell proliferation upon depletion or readdition of $\mathrm{CD}_{14}{ }^{+}$cells from patients' peripheral blood mononuclear cells (PBMCs) (Supplemental Figure 1F). Interestingly, $\mathrm{CD} 14^{+}$cells from patients retain the potential to acquire EV-MDSC features, measured as HLA-DRA downregulation in the presence of melanoma EVs (Figure 1D). Furthermore, we could ascertain the induction of functional EV-MDSCs in an autologous setting, i.e., with EVs purified from CM of a patient's melanoma cell line, which we incubated with the patient's monocytes isolated from PBMCs; these EV-MDSCs were able to inhibit autologous $\mathrm{T}$ cell proliferation (Figure 1E).

Parallel experiments have been performed in a murine setting with EVs released by the Ret melanoma cell line established from skin melanomas developed by RET transgenic mice (ret-EVs) (26). ret-EVs converted BM IMCs into MDSCs in vitro, boosting NO, inducible NOS (iNOS), and arginase-I expression and immunosuppressive activity on $\mathrm{T}$ cell proliferation (Supplemental Figure 2, A-C). Upon i.p. administration to naive C57BL/6 mice, fluorescently labeled EVs interacted with $\mathrm{Gr}^{+} \mathrm{CD} 11 \mathrm{~b}^{+}$IMCs and, to a lesser extent, with $\mathrm{F} 4 / 80^{+} \mathrm{Gr} 1^{\text {neg }}$ macrophages in the spleen, BM, and LNs (Supplemental Figure 2D). Repeated administration of ret-EVs led to a significant increase only in the frequency of $\mathrm{Gr} 1^{+} \mathrm{CD} 11 \mathrm{~b}^{+} \mathrm{Ly} 6 \mathrm{C}^{\text {hi }} \mathrm{M}-\mathrm{MDSCs}$, detectable in the BM (Supplemental Figure 2E). 
A
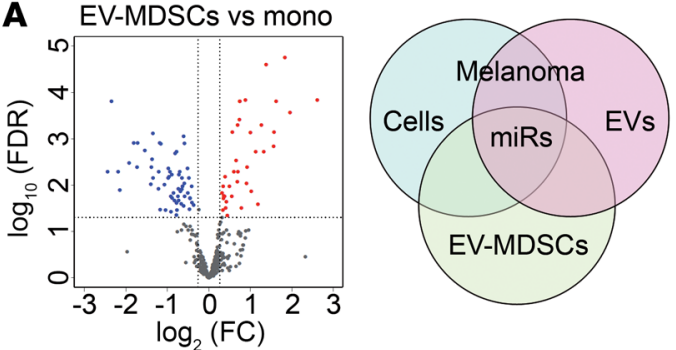

B
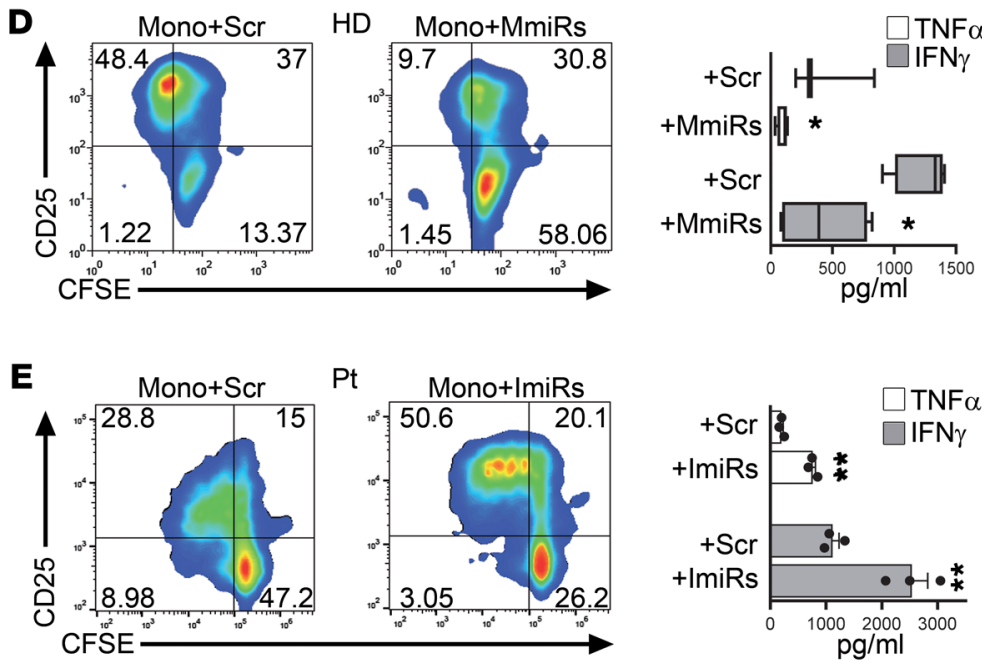

$\mathbf{F}$

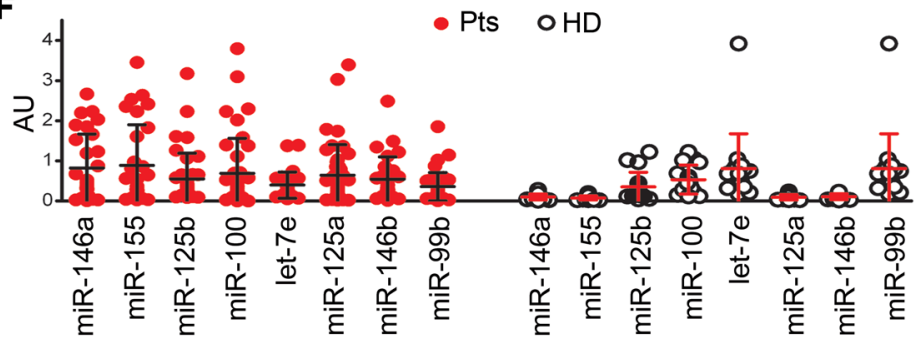

Top miRs regulating repressed genes $\operatorname{miR}-99 \mathrm{~b}$ miR-146b $\rightarrow-1$ miR-125a $\bullet-1$ let-7e $\bullet-0$ miR-100 $\operatorname{miR}-125 b$ $\operatorname{miR}-155$ miR-146a 1,
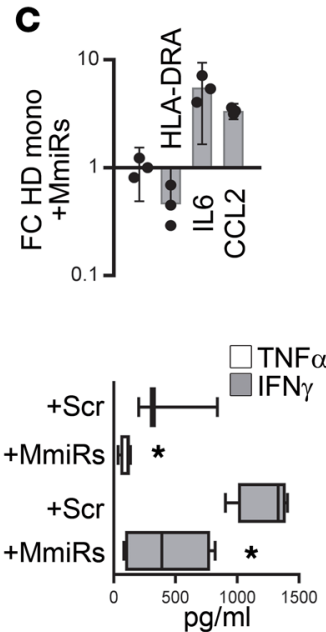

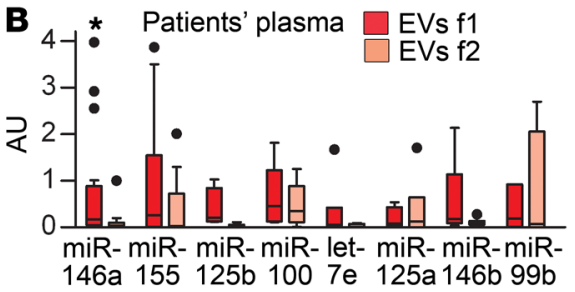
FC EV-MDSCs

tant at $118,000 \mathrm{~g}$ ), as indicated by transmission electron microscopy (TEM) and bead-calibrated flow cytometry (Supplemental Figure 3B). Interestingly, f1 EVs from melanoma patients' plasma downregulated HLA-DRA at RNA levels in HD CD14 ${ }^{+}$monocytes upon in vitro coculture, suggesting the presence of EV-MDSC-converting vesicles potentially derived from tumor cells (Figure 1G). This effect was less frequent with $\mathrm{f} 2 \mathrm{EVs}$ from melanoma patients, which, in contrast, induced an increase of HLA$D R A$ expression in HD monocytes. The upregulation of $H L A-D R A$ at the RNA level could be ascribed to monocyte activation, possibly due to the presence of stimulating EVs in $\mathrm{f} 2$ plasma fractions, likely originating from antigen-presenting cells (APCs), similarly to what we observed with $\mathrm{f} 1$ and $\mathrm{f} 2$ from plasma of HDs (Figure 1G). Reports have indeed shown that plasma exosomes can activate monocytes by inducing the upregulation of HLA-DR and CD86 expression in healthy and pathological conditions $(27,28)$.

Similarly to normal monocytes, monocytes from melanoma patients downregulated their HLA-DRA expression upon coculture with autologous plasma f1 and f2 EVs, accentuating the potential relevance of this phe-

Since tumor EVs can circulate in body fluids together with EVs originating from immune and other cells, we tested to determine whether plasma EVs from advanced melanoma patients could also promote the EV-MDSC phenotype in vitro. EV screening of unprocessed plasma by nanoparticle tracking analysis (NTA) showed that patients' samples contained EVs in increased numbers and mean sizes as compared with those from HDs. Notably, the size of EVs directly correlated with the frequency of circulating MDSCs in matched peripheral blood samples, suggesting a potential link to the disease (Figure $1 \mathrm{~F}$ and Supplemental Figure 3A). To obtain vesicles for functional studies, we applied a 2-step centrifugation protocol aimed at enriching for larger (f1, obtained at $16,500 \mathrm{~g}$ ) versus smaller EVs (f2, obtained with subsequent centrifugation of f1 supernanomenon in vivo. Of note, EV-MDSCs generated with autologous plasma f1 and $\mathrm{f} 2 \mathrm{EVs}$ displayed an increased suppressive effect on $\mathrm{T}$ cell proliferation with respect to unconditioned monocytes (Figure $1 \mathrm{H})$. The actual presence of melanoma EVs in patients' plasma could be confirmed by protein analysis, showing that, in contrast with HD, both f1 and f2 EVs of the patient expressed the gp100 melanoma marker, with a tendency to accumulate in $\mathrm{fl}$. These data may contribute to explaining the downregulation of $H L A$ $D R A$ in HD monocytes that we observed predominantly with $\mathrm{f} 1$ plasma EVs from melanoma patients, as shown in Figure 1G. An increase in $\mathrm{f} 1$ could also be assessed for the EV markers Rab5B, CD63, and CD49b/VLA-2 integrin, while both fractions showed similar levels of the LAMP-2 EV marker (Figure 1I). 
A $\square$ EVs from ImiR-cells
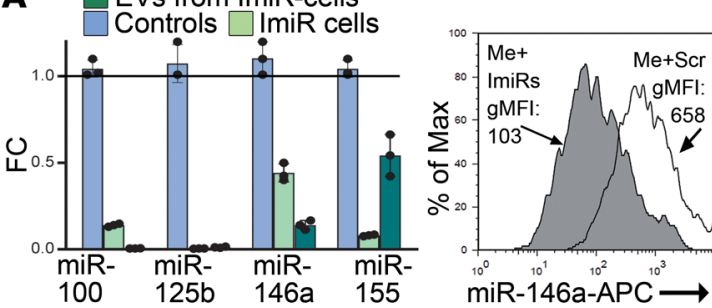

C
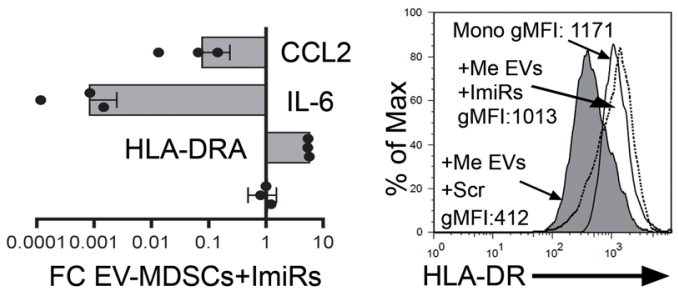

$\mathbf{E}$

Me EVs
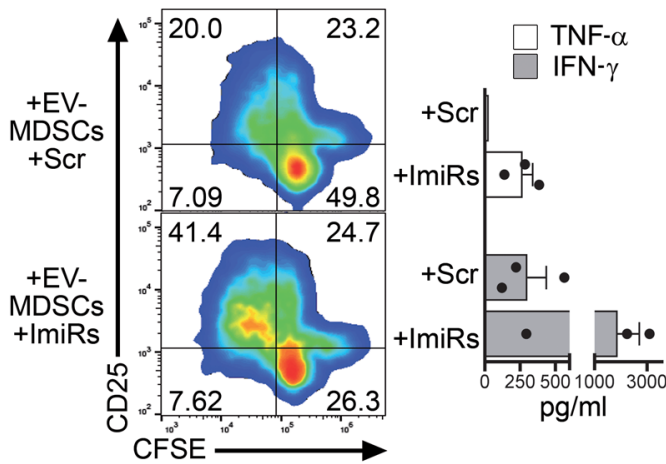

B

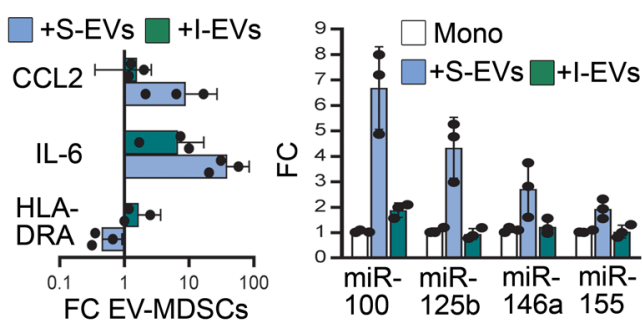

D

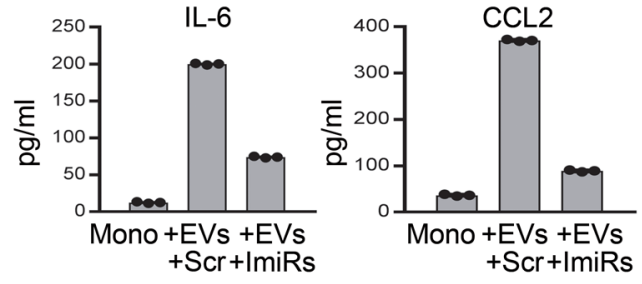

$\mathbf{F}$ Autologous Me EVs

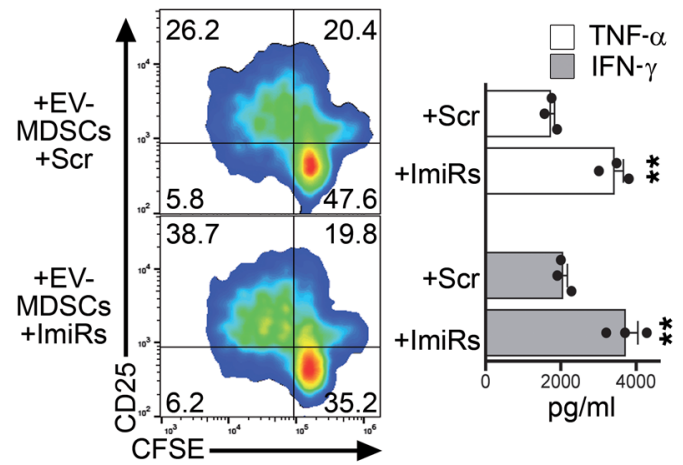

Figure 4. Inhibition of MDSC-miRs rescues monocytes from the acquisition of a suppressive phenotype. (A) Effect of transfection with miR inhibitors or scrambled control (Scr) on MDSC-miR expression in INT12 melanoma cells and respective EVs (left panel). Reduced expression of miR-146a in silenced melanoma cells (Me) was confirmed by flow cytometry using an APC-fluorescent SmartFlare probe (right panel). (B) EVs derived from miR-silenced melanoma cells (+I-EVs) impaired the induction of the EV-MDSC phenotype compared with EVs derived from scrambled control cells (+S-EVs) (left panel); MDSC-miR expression was reduced in monocytes treated with I-EVs compared with those treated with S-EVs (right panel). (C) Effect of transfection with miR inhibitors or scrambled control of HD monocytes cultured in the presence of melanoma EVs, as evaluated by qPCR and flow cytometry, and (D) IL-6 and CCL2 release. (E) Loss of immunosuppressive activity of EV-MDSCs, generated from HD in the presence of miR inhibitors or scrambled control, on activated CFSE-labeled T cells, as evaluated by flow cytometry (left panel) and cytokine release (right panel), and (F) loss of immunosuppressive activity of EV-MDSCs generated from a patient with autologous melanoma-derived EVs. Percentages of CD25 and CFSE expression are indicated. Cells transfected with scrambled control were used as a calibrator $(\mathbf{A}-\mathbf{C})$. $P<0.05$, paired Student's $t$ test $(\mathbf{A}-\mathbf{C}) ; P<0.001,1$-way ANOVA (D). ${ }^{*} P<0.05$, ${ }^{* *} P<0.01$, paired Student's $t$ test (E, F). Experiments were repeated twice and performed in triplicate (A-D, F). Data are representative of $2 \mathrm{HD}$ tested (E).

The transcriptional regulation of EV-MDSCs involves miR modulation. We next investigated the molecular mechanisms leading to the in vitro induction of EV-MDSCs. Genome-wide transcriptional analysis revealed 662 genes, 514 upregulated and 148 downregulated (fold change $[\mathrm{FC}]>|2|, \mathrm{FDR}<0.05$ ), as differentially expressed in EV-MDSCs with respect to untreated monocytes (Supplemental Table 1). Gene Ontology (GO) enrichment analysis indicated that most of the upregulated genes were involved in various immune cell functions, including inflammatory response, apoptosis signaling pathways, signal transduction regulation, and wound healing (Supplemental Table 2). Notably, the expression pattern of bona fide MDSC hallmark genes in the human setting is detectable in EV-MDSCs at the transcriptional level. We also identified key chemokines for protumor myeloid cell functions and hematopoietic stem cell maintenance, includ- ing CCL1, CCL22, CXCL5, CCL7, CSF1, and PPBP/CXCL7, among the top hits. Several transcriptional factors were upregulated, including HIF1, mTOR, NOTCH1, NFKB2, RUNX1, MYC, ETS1, factors involved in STAT3 downstream signaling pathways (MYC, CCND1, and MCL1), and different SMAD genes, most of which have been reported to be involved in MDSC functions (29-31). Genes involved in increased survival and antiapoptotic effects (e.g., BCL2 and CDKN2A) were enhanced, together with some molecular hallmarks of murine MDSCs, including S100A8 and MMP9 (Figure 2A). Notably, approximately 10\% of genes differentially expressed in EV-MDSCs were associated with EMT, as revealed by analysis of the overlap in a list of EMT-related genes; this analysis included an EMT database (32), gene sets associated with EMT and/or wound healing in the IPRES signature (5), and genes common to mesenchymal and myeloid cells (33) (Figure 
A

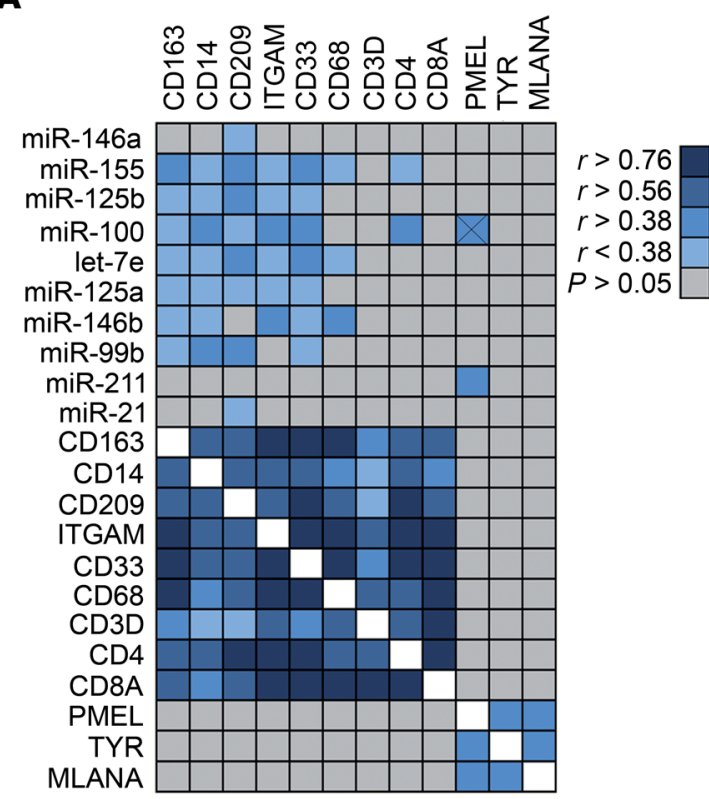

B
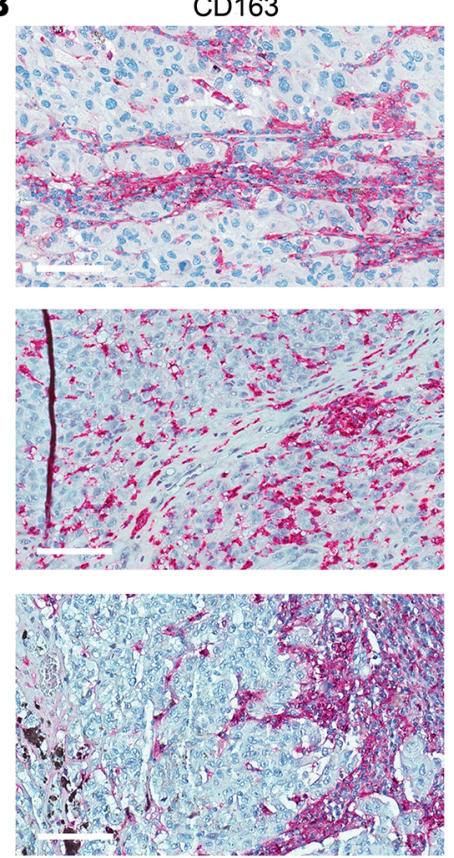
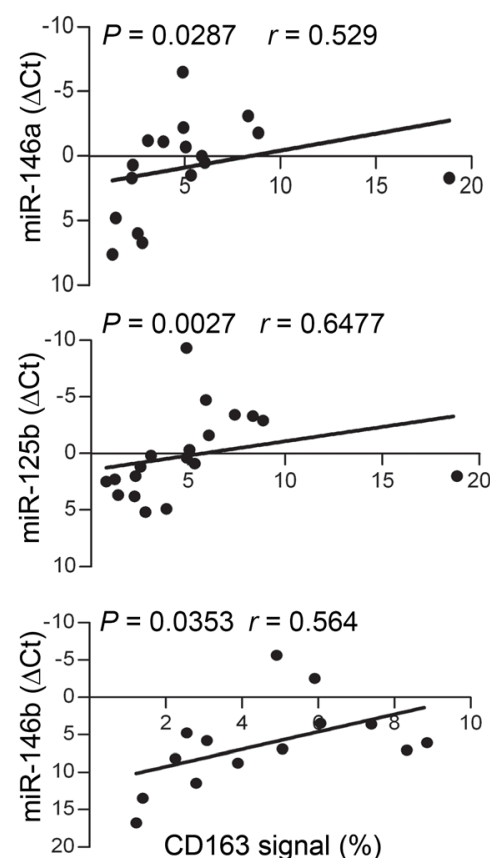

C
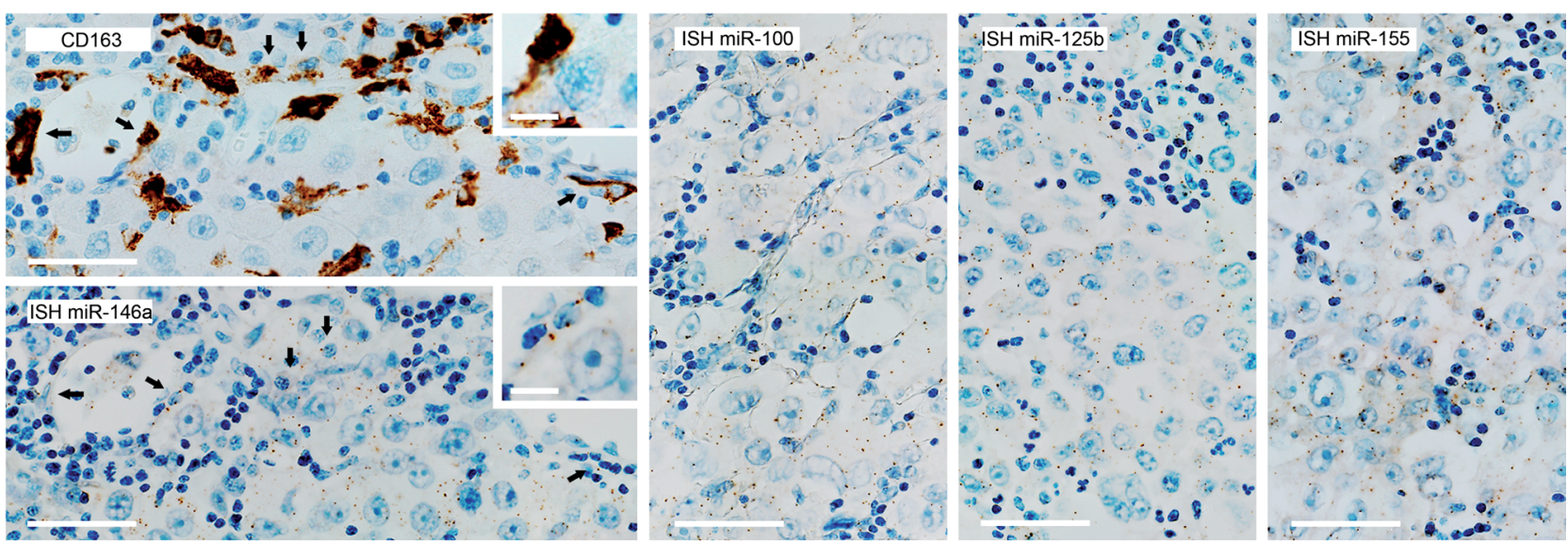

Figure 5. MDSC-miRs are expressed in tumor specimens and monocytes of melanoma patients. (A) Correlation matrix of the expression levels of MDSC-miRs and CD163, CD14, CD209, ITCAM, CD33, CD68, CD3D, CD4, CD8A, PMEL, TYR, and MLANA in metastatic melanoma specimens ( $n=58) ; P M E L, T Y R, ~ M L A N A$, miR-21, and miR-211 are used as unrelated controls. $r$ values from univariate Spearman's analysis in correlations with $P<0.05$. (B) Immunostaining of CD163 infiltrate in melanoma (left panel) and correlation of the quantified CD163 signal with the expression levels of MDSC-miRs (right panel), as determined by qPCR $(n=20)$. (C) ISH images showing the expression of miR-146a, miR-100, miR-125b, and miR-155 in representative tumor sections. miR signals appear as brown dots localized both in CD163+ infiltrating cells and in melanoma cells. Scale bars: $100 \mu \mathrm{m}$. Representative images are shown in B and C.

2B). The modulation of critical genes, including the downregulation of HLA-DRA, HLA-DPA1, CD1D, and TLRs (TLR4, TLR5, $T L R 7)$ and the upregulation of CD274/PD-L1, TNFRSF8/CD30, genes promoting angiogenesis and metastasis (PTGS2 and VEG$F A$ ), and genes controlling cell survival and proliferation (BCL2A1 and $C D K N 2 A)$, have been confirmed by quantitative real-time PCR (qPCR) analysis (Figure 2C).

The CIBERSORT assessment of transcriptome data (34) shows a significant enrichment of the MO fraction of nonpolarized monocytes (Supplemental Figure 4) rather than M1 and M2 polarized macrophage fractions (35). In silico analysis of EV-MDSCregulated genes also indicated a consistent overlap with an MDSC gene list described in colon tumor tissue data sets (36). To con- firm the overlapping features of our EV-MDSCs with myeloid cells from cancer patients, we compared the transcriptomic profile of EV-MDSCs with those of monocytes sorted from the blood of patients with different tumor types (37-39) and found a significant enrichment of the overexpressed genes (Figure 2D).

Monocyte skewing to EV-MDSCs also showed changes in $\mathrm{miR}$ expression. Via profiling, $98 \mathrm{miRs}$ were identified as differentially expressed between monocytes and EV-MDSCs (FC $|1.2|$, FDR $<0.05) ; 34$ were differentially expressed, with a FC of $\geq|2|$, including miRs, such as miR-146a, miR-125, and miR-155, described as being involved in the regulation of classical and alternative myeloid cell activation (Figure 3A and Supplemental Table 3) (40-42). Integrative analysis of miRs and gene expres- 

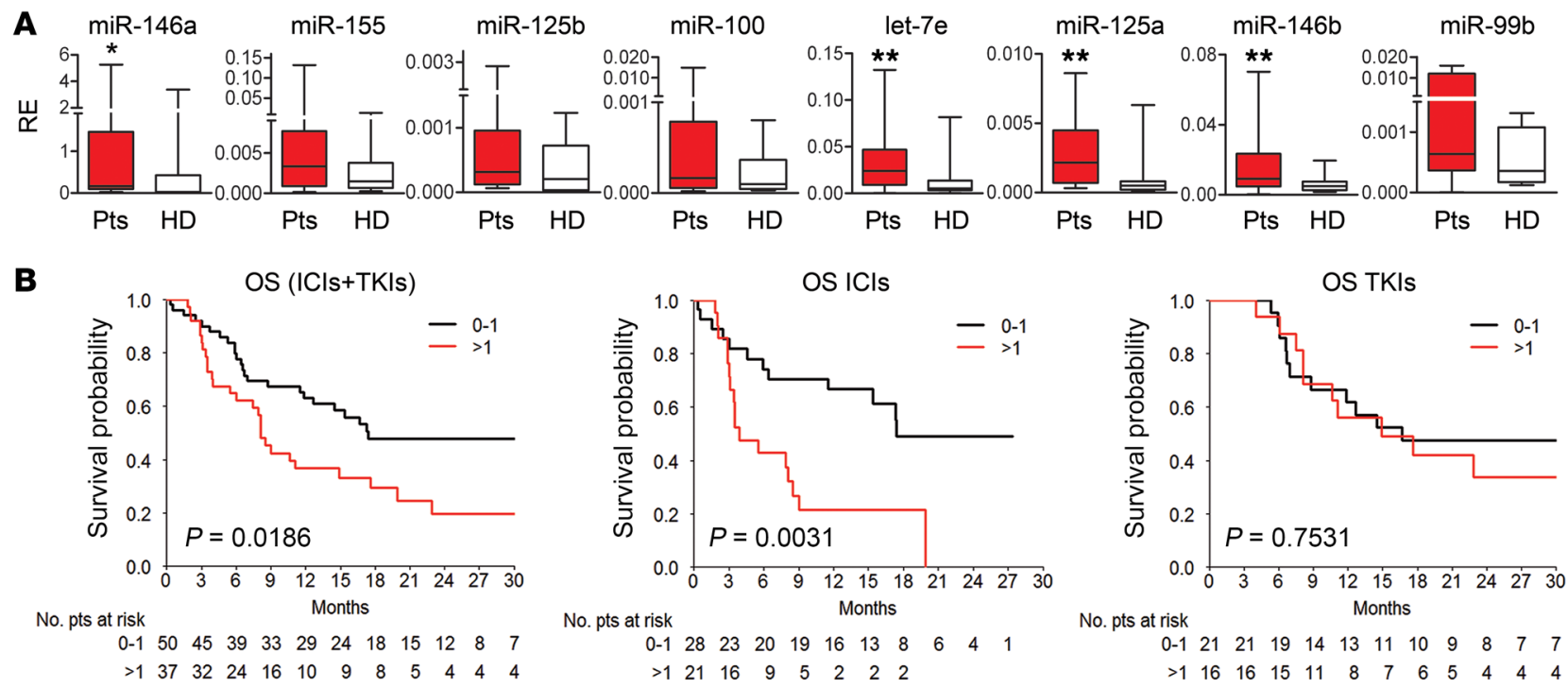

Figure 6. MDSC-miRs are enriched in plasma from melanoma patients and associate to resistance to immunotherapy. (A) MDSC-miR detection in the plasma of patients (Pts) and HD ( $n=20$ /group). miR expression levels were normalized using ath-miR-159a as reference miR. RE, relative expression. ${ }^{*} P<0.05$; ${ }^{* *} P<0.01$, Mann-Whitney $U$ test. (B) OS of metastatic melanoma patients based on the expression levels of MDSC-miRs in plasma samples obtained at baseline of therapy, assessed by multivariable index score approach and AIM, in the global population ( $n=87$; left panel) and in the subsets of patients receiving immunotherapy $(I C I s, n=49)$ or targeted therapy (TKIs, $n=37)$. One patient was excluded from the latter because of receiving chemotherapy. Patients with low scores ( $0-1$; showing 0 or only 1 increased miR) had a significantly better OS compared with patients with high scores (>1; having 2-5 increased miRs) only if receiving ICls. Kaplan-Meier survival curves with log-rank $P$ values are shown.

sion profiling data revealed regulatory networks governing key myeloid cell functions, including the chemotaxis, adhesion, differentiation, and recruitment of phagocytes, macrophages, and APCs (Supplemental Figure 5).

miRs induce the EV-MDSC phenotype in $C D 14^{+}$monocytes. Since EVs are known miR carriers (43-45), we hypothesized that EV-mediated miR cargo is involved in the process of in vitro EV-MDSC conversion. Profiling enabled us to identify 104 miRs detectable in both melanoma EVs and matched tumor cells (Supplemental Table 4). By crossing this set of miRs with those overexpressed in EV-MDSCs and associated with gene downregulation, we selected the top upregulated miRs - namely, miR-146a, miR155, miR-125b, miR-100, let-7e, miR-125a, miR-146b, and miR$99 \mathrm{~b}$ - as MDSC-associated miRs for subsequent studies (Figure 3A; Supplemental Table 5). The evaluation of these miRs in plasma EV fractions $\mathrm{f} 1$ and $\mathrm{f} 2$ of melanoma patients showed that they were all detectable and that 5 out of 8 miRs (miR-146a, miR-146b, let-7e, miR-99b and miR-125b) were enriched in the f1 EV fraction, while the others appeared equally distributed (Figure 3B). Similarly, melanoma cell line-derived EVs showed comparable levels of MDSC-miRs in $\mathrm{fl}$ and $\mathrm{f} 2$, compatible with the release of larger and smaller EVs by melanoma cells in vitro. Of note, the miR content associated with the induction of EV-MDSCs, as shown by HLADRA downregulation in HD monocytes (Supplemental Figure 6). The contribution of MDSC-miRs in MDSC conversion was then explored with the help of corresponding mimics transfected into HD monocytes. Similarly to EV-MDSCs, miR-transfected monocytes displayed downregulation of HLA-DRA and the induction of IL6 and CCL2 expression (Figure 3C). In line with their MDSC nature, these cells also exerted suppressive activity on $\mathrm{T}$ cells, as suggested by the impairment of proliferation, CD25 expres- sion, and IFN- $\gamma$ and TNF- $\alpha$ secretion in T cell receptor-triggered (TCR-triggered) autologous T cells (Figure 3D). Conversely, the transfection of melanoma patient-derived monocytes with miR inhibitors led to their functional recovery (Figure 3E), indicating that MDSC-miRs play a role in sustaining myeloid cell-related protumor effects. The potential link of MDSC-miR expression and the accumulation of MDSCs in melanoma patients' peripheral blood was also supported by a significantly higher expression of 6 out of 8 MDSC-miRs in the patients' samples, which we observed upon comparing the levels of MDSC-miRs in $\mathrm{CD} 14^{+}$cells sorted from PBMCs of HD with those of metastatic stage IIIC-IV melanoma patients at treatment baseline (i.e., at $\geq 1$ month apart from any systemic therapy) (Figure 3F).

We next assessed the contribution of direct MDSC-miR transfer from a tumor to monocytes via EVs with the aid of EVs derived from melanoma cells silenced for miR-146a, miR-100, miR-125b, and miR-155. miR-devoid EVs lost the ability to convert monocytes into EV-MDSCs in terms of HLA-DRA downmodulation, CCL2 and IL6 induction, and MDSC-miR upregulation (Figure 4 , A and B). Similarly, the contemporaneous inhibition of these MDSC-miRs by adding miR inhibitors to melanoma EV-exposed monocytes from HD abrogated the acquisition of the EV-MDSC phenotype as well as suppressive activity on $\mathrm{T}$ cell activation and cytokine production (Figure 4, C-E). Of note, the addition of miR inhibitors to a patient's monocytes conditioned with EVs deriving from the autologous melanoma cell line also restored $\mathrm{T}$ cell activity and cytokine release (Figure $4 \mathrm{~F}$ ).

Taken together, these results show that MDSC-miRs display an increased expression in monocytes of patients, circulate within EVs in patients' plasma, and condition myeloid cells via EV-mediated transfer. 
MDSC-miRs are enriched in correlation with myeloid cell accrual. The relevance of MDSC-miRs was next investigated ex vivo in human melanoma specimens. As expected, we detected high miR146a, miR-155, miR-125b, miR-100, let-7e, miR-125a, miR-146b, and miR-99b levels in subcutaneous, visceral, and LN metastases from stage III-IV melanoma patients $(n=58)$ by qPCR, confirming the expression of these miRs by this tumor type (Supplemental Figure 7). Interestingly, MDSC-miRs correlated with myeloid markers CD163, CD14, CD209, CD68, ITGAM (CD11b/Mac-1), and $C D 33$ detected in the same samples, but not with T cell markers $C D 3, C D 4$, and $C D 8$ or with tumor-related markers $P M E L$, TYR, MLANA, miR-21, and miR-211; the only exceptions were miR-155 and miR-100, which correlated with CD4 (Figure 5A and Supplemental Table 6). Multivariate statistical analysis showed a significant correlation between the MDSC-miR cluster and the expression of all myeloid markers $(P=0.0062, r=0.5491)$. Additionally, the correlation of CD14 and CD163 expression with the miR-146a, miR-100, miR-125b, and miR-155 clusters was also significant $(P=0.0455, r=0.2088)$. The association of MDSC-miRs with infiltrating myeloid cells at the RNA level was confirmed by the correlation of miR-146a, miR-125b, and miR-146b with the number of $\mathrm{CD} 163^{+}$cells in tumor sections (Figure 5B). Indeed, in situ hybridization revealed that miR-146a, miR-100, miR-125b, and miR-155 expression was localized in both $\mathrm{CD}_{163}{ }^{+}$and melanoma cells (Figure 5C). These data suggest that these miRs might be indicators of MDSC activity at the tumor site.

MDSC-miRs are enriched in the plasma of melanoma patients and cluster with resistance to immunotherapy. To assess MDSCmiRs in the circulation, we measured their expression levels in plasma obtained from melanoma patients with advanced disease (stage IIIC unresectable and stage IV) $(n=20)$ and from a group of age- and sex-matched HD $(n=20)$. The analysis showed increased levels of all MDSC-miRs in the patients, with statistically significant differences shown for miR-146a, let-7e, miR-125a, and miR146b (Figure 6A). Notably, the expression levels of the studied miRs appeared more intercorrelated in patients than in HD (data not shown), further indicating an association with disease.

To test the potential for using MDSC-miRs as peripheral blood-based markers, we conducted a retrospective analysis in a set of plasma samples obtained from metastatic melanoma patients $(n=87)$ receiving either ICIs (ipilimumab or nivolum$\mathrm{ab)}$ or targeted therapy (tyrosine- kinase inhibitors [TKIs]; vemurafenib or the combination of dabrafenib and trametinib). Enrolled patients included 49 and 37 patients in the ICI and TKI groups, respectively (Supplemental Table 7). The expression levels of MDCS-miRs in the baseline samples were analyzed and correlated with OS and progression-free survival (PFS), both singly and jointly, in the latter case via a machine learning tool known as adaptive index modeling (AIM). By estimating the joint contribution of the 5 miRs showing an association with disease outcomes in univariable assessments (let-7e, miR-125a, miR-99b, miR-146b, and miR-125b), we were able to divide patients into 5 groups on the basis of an index score comprising the number of miRs showing increased expression, as detailed in Methods. Regarding OS, by pooling low scores ( $0-1$; consisting of 0 or only 1 increased miR) versus high scores ( $>1$; having 2 to 5 altered miRs), we observed that the low scores were associated with significantly better OS than were the high scores (Figure 6B). A comparable trend was observed when PFS was analyzed (Supplemental Figure 8). Most importantly, when these data were divided by the type of therapy, the difference in OS between low and high scores was much more evident, with a $P$ value of 0.003 , for ICI-treated patients; no difference was detected for TKI-treated patients. Comparable data were observed for PFS; however, the curves for ICI patients were less divergent than were those for OS due to the known minor impact of ICIs on PFS (2). These data clearly indicate that our MDSCmiRs may represent predictive markers of the response to or the benefit of ICIs in advanced melanoma patients.

\section{Discussion}

In the present work, we discovered a set of miRs that associates with the phenotypic and functional features of M-MDSCs in melanoma patients. These miRs are detected in plasma as associated with EVs, in blood monocytes, and in tumor biopsies, as correlated with the frequency of altered myeloid cells. Most importantly, higher circulating levels of these miRs cluster with shorter PFS and OS in patients receiving ipilimumab and nivolumab, but not in those treated with BRAF/MEK inhibitors. These latter findings, if prospectively validated, pave the way for the development of what, to our knowledge, represents the first predictive peripheral blood biomarker of resistance to ICIs in cancer. The assessment of MDSC-miRs would make use of the concept of liquid biopsy (46), which has traditionally focused on genetic tumor features (47), but surely could be implemented with information from the systemic environment, including host immunity (48).

The evidence that factors associated with myeloid-related immune suppression might influence disease outcome in melanoma patients is not unexpected, as higher MDSC frequency is a constant in poor disease prognosis, even during ICI administration (14-17). The true predictive role of the MDSC-miRs here identified might instead reflect specific functional properties that MDSCs could exhibit once immune responses are unleashed by the ICIs (29). This hypothesis is in line with preclinical data showing that tumor resistance to PD-1 or CTLA4 blockade can be reverted by myeloid cell depletion $(49,50)$.

Major evidence, albeit primarily in murine models, indicates a central role of miR-mediated posttranscriptional regulation in MDSC differentiation and function $(40,51)$. Via in vitro studies on monocyte conditioning, we found that hallmarks of human MDSCs, i.e., downregulation of HLA-DR, secretion of IL-6, TGF- $\beta$, and CCL2, and inhibition of T cell proliferation and function $(9,10)$, can be reconstituted by in vitro cotransfection of the 8 MDSC-miRs; this finding held true even when the requirement was decreased to 4 miRs (miR-146a, miR-100, miR-125b, and miR-155) in some donors (data not shown). The chance of capturing such a complex regulatory scenario likely stems from the use of tumor EVs for MDSC generation. Indeed, EVs are known to carry distinct sets of miRs and to deliver them into target cells for the induction of specific functions, a key mechanism of intercellular crosstalk (52). In the context of cancer, transformed cells exploit EV-mediated miR transfer to mold host tissues by triggering angiogenesis, EMT, invasion and metastasis, immune escape, and multidrug resistance $(23,43)$. Here, we provide experimental proof that melanoma EVs are embedded with a set of miRs 
ready to convert monocytes into bona fide MDSCs upon direct transfer. As this process is mimicked by ret-EVs injected in vivo into $\mathrm{C} 57 \mathrm{BL} / 6$ mice (Supplemental Figure 2E), it is reasonable to hypothesize that MDSC accumulation in melanoma patients might also involve in vivo myeloid conditioning by tumor EVs. The ability of these nanoparticles to distribute in body fluids (20) and reach the BM as well as other immunoregulatory sites could then account for the alteration of myelopoiesis, as depicted by our mouse studies as well as by previous preclinical data (18).

An in vitro tumor-mediated conversion of differentiated monocytes to MDSC-resembling cells was already reported by others (53). This process can be explained by the high plasticity of monocyte differentiation that cancer can arrest and shift toward immunoregulatory/suppressive mediators, as originally described for sepsis and cardiovascular diseases (54). This evidence supports the recently introduced hypothesis that cancer-associated myeloid dysfunctions could stem not only from progenitor conditioning in the $\mathrm{BM}$, but also from the peripheral reprogramming of circulating differentiated monocytes and neutrophils $(55,56)$.

Most of the miRs here identified are involved in myeloid cell differentiation and polarization by participating in pathways often associated with cancer-related immunosuppression (57-59). For instance, miR-146a, one of the most studied myeloid miRs (40), is reported to serve as negative feedback in the TLR4-mediated activation of NF- $\kappa \mathrm{B}$-related genes (60) and to promote M2 polarization in both humans and mice (42). miR-155, in addition to its key role in T cell responses (61), is the top upregulated miR in GM-CSF- and IL6-induced MDSCs and is involved in STAT3 activation via SHIP-1 and PTEN targeting (62). This miR has also been shown to facilitate tumor growth by promoting MDSC accrual, survival, and function through SOCS1 inhibition (63-66). Similarly, miR-125b and miR-125a have been implicated in monocyte differentiation toward immunosuppressive phenotypes $(40,42)$ via a pathway involving Lin28A (67), a key factor in hematopoietic stem cell growth and survival (68). miR-125b is produced with miR-100 from a common primary polycistronic transcript; miR-99b, let-7e, and miR-125a are produced via a similar mechanism (69), which explains their coordinated expression in tumor biopsies and in circulating monocytes from melanoma patients (Figure $5 \mathrm{~A}$ and Figure $3 \mathrm{~F}$ ). The miR-99b/let-7e/miR-125a miR cluster was also shown to play a role in the mechanisms that stabilize STAT-3 activity in tolerogenic APC (70). Specific insights into the pathways targeted by MDSC-miRs derive from matching their predicted gene panels with the transcriptional profile of EV-MSDCs, an analysis that provides key information about the vital processes of cancer-related myeloid dysfunctions involving chemotaxis, adhesion, and differentiation, with nodes including expected and previously unidentified patterns (Supplemental Figure 5).

Overall, our study sheds light on the poorly defined patterns of human MDSC activity, providing strong evidence about the role of defined miRs in driving cancer-related myeloid cell reprogramming. The involvement of MDSC activation and expansion in limiting the clinical efficacy of ICIs is also strengthened by our results, pointing to the need for drugs for the correction of myeloid dysfunctions (51) to overcome immunotherapy resistance in melanoma patients. Finally, the evaluation of MDSC-miR plasma lev- els holds promise as a potential biomarker to assess and monitor systemic immunosuppression in cancer patients, possibly predicting their chance of responding to ICIs.

\section{Methods}

Human studies. Peripheral blood samples from fasting patients and HD were processed within 2 hours for plasma collection and PBMC isolation and storage (11). PBMCs from stage III-IV patients obtained before surgery or medical treatment were studied for MDSCs by immunophenotyping, and sorted $\mathrm{CD} 14^{+}$cells were assayed for cytokine, miR expression, and MDSC conversion and suppressive function. Plasma from stage IIIC-IV patients at baseline prior to any medical treatment was studied for circulating EVs and miRs. Metastatic tumor specimens obtained from stage IIIC-IV melanoma patients were snap-frozen and processed for RNA extraction and analyses.

Mouse studies. C57BL/6 mice were purchased from Charles River Laboratories. In vivo and ex vivo interaction studies investigating the effects of ret melanoma EVs on myeloid cells involved the i.p. injection of $200 \mu \mathrm{g}$ EVs, labeled with Cell Proliferation Dye eFluor 670 (Thermo Fisher Scientific) for interaction, or unlabeled EVs injected 3 times every 48 hours. Four days after the last injection, the animals were sacrificed to collect the BM and spleen cells for flow cytometry evaluation. CD11b $\mathrm{Gr}^{+}$ IMCs $\left(10^{5}\right)$ were cultured with $5 \mu \mathrm{g}$ EVs overnight. NO production was measured by Griess Reagent (Promega), and arginase-I and iNOS expression were measured by flow cytometry. Treated IMCs were washed twice by centrifugation to remove EVs and coincubated with CFSE-labeled T cells stimulated with CD3/CD28 mAbs (T cells/IMCs =1:1).

Cell cultures. Melanoma cell lines were generated in our laboratory from human melanoma specimens (71). The ret mouse melanoma cell line was established from the skin tumors of ret transgenic mice (26). Mycoplasma contamination was tested periodically.

Isolation of EVs. According to a standardized protocol optimized for large-scale EV production, the supernatant of $10^{9}$ human melanoma cells, cultured for 72 hours in RPMI 1640 medium containing 10\% EV-depleted FCS (obtained by centrifuging FCS 2 hours at 118,000 g), $2 \mathrm{mM}$ L-glutamine, and $200 \mathrm{U} / \mathrm{ml}$ penicillin/streptomycin, was centrifuged at $300 \mathrm{~g}$ for 10 minutes, $0.22 \mu \mathrm{m}$ vacuum filtered (Millipore), and ultracentrifuged (Sorvall WX Ultra 100, Thermo Fisher Scientific) at $100,000 \mathrm{~g}$ for 2 hours at $4^{\circ} \mathrm{C}$. Subsequently, collected EVs were suspended in PBS and washed by ultracentrifugation (1 hour, 118,000 $\mathrm{g}$, $4^{\circ} \mathrm{C}$ ). After protein quantification (Bradford Protein Assay, Bio-Rad), aliquots were frozen at $-80^{\circ} \mathrm{C}$ until further use. Ret murine melanoma EVs were purified from 48 hours serum-free CM of ret cells by sequential centrifugation (300 $g$ for 10 minutes, 4,000 $g$ for 20 minutes), $0.22 \mu \mathrm{m}$ vacuum filtering, and ultracentrifugation at 100,000 $g$ for 4 hours at $4^{\circ} \mathrm{C}$. Further processing was analogous to the process for EVs from human melanoma cell lines. Plasma samples were obtained from EDTA blood samples after centrifugation at $3000 \mathrm{~g}$ for 10 minutes, followed by plasma centrifugation at $3000 \mathrm{~g}$ for 10 minutes. For EV isolation, $500 \mu \mathrm{l}$ plasma diluted 1:8 times with $0.1 \mu \mathrm{m}$-filtered PBS was centrifuged at $16,500 \mathrm{~g}$ for 10 minutes to obtain the fraction $1 \mathrm{EV}$ pellet, and the supernatant was centrifuged at 118,000 $\mathrm{g}$ for 1.5 hours to sediment the fraction $2 \mathrm{EVs}$. The same protocol was also applied to isolate $\mathrm{f} 1$ and $\mathrm{f} 2$ human melanoma EVs from $\mathrm{CM}$.

NTA. The size distribution and concentration of EVs purified from CM of melanoma cells (5 $\mu$ g diluted 1:10,000) and of EVs in unprocessed plasma samples (5 $\mu$ l diluted 1:10,000) or cell-depleted CM 
of melanoma cells (dilution 1:2) were determined using an LM10-HS NanoSight instrument and evaluated with NTA software (Malvern Instruments). Preparations were analyzed 5 times for 30 seconds.

TEM. Freshly isolated plasma EV preparations were fixed with $4 \%$ paraformaldehyde and deposited on Formvar carbon-coated nickel grids. Grids were doubly stained with uranyl acetate and lead citrate and examined by TEM (CM 10 Philips; FEI).

Western blot analysis. Proteins obtained from purified EVs and cells were separated on $4 \%$ to $12 \%$ Bis-Tris precast gels (Invitrogen), transferred to nitrocellulose membranes (Amersham), and incubated with Abs. Reactive proteins were visualized using HRP-conjugated secondary Abs (Amersham) and enhanced chemiluminescence (Amersham, SuperSignal). The list of Abs used is provided in Supplemental Table 8.

Flow cytometry. To perform immunophenotyping studies, human and mouse cells were incubated with specific fluorochrome-conjugated Abs or isotype controls, provided in Supplemental Table 8, washed, and analyzed. Flow cytometry of EVs from melanoma cells was performed using sulfate/aldehyde $5 \mu \mathrm{m}$ latex beads (Thermo Fisher Scientific) (72). A SmartFlare detection probe for miR-146a was used according to the manufacturer's instructions (Merck Millipore). Samples were evaluated using a FACSCalibur (BD Biosciences) and a Gallios or Cytoflex flow cytometer (Beckman Coulter) and FlowJo software (TreeStar Inc.). Morphology of plasma EV fractions was assessed by acquisition with the Gallios flow cytometer (Beckman Coulter) after calibration with 200, 500, and $1000 \mathrm{~nm}$ beads (Polysciences).

Monocyte studies. Monocytes (purity $>95 \%$ ) were purified from buffy coats of HD and from patients' PBMCs by sorting with antiCD14 ${ }^{+}$beads (MACS, Miltenyi Biotech) according to the manufacturer's instructions. For the generation of EV-MDSCs, CD14 ${ }^{+}$cells $(0.3$ $\times 10^{6}$ in $\left.300 \mu \mathrm{l}\right)$ were cocultured with melanoma EVs $(50 \mu \mathrm{g})$ in complete medium supplemented with $10 \%$ EV-depleted FCS for 24 hours. For the transfection of miR inhibitors or miR mimics (QIAGEN), 50 $\mathrm{nM}$ oligos in HiPerfect transfection reagent (QIAGEN) were admixed with monocytes or melanoma cells for 4 hours before the addition of FCS-containing medium. For miR inhibition in melanoma cells, EVs were isolated from CM 3 days after transfection with inhibitors, as described above. For proliferation studies, human $\mathrm{T}$ cells were stained with CFSE (Thermo Fisher Scientific) prior to stimulation with antiCD3 and anti-CD3/CD28 mAbs and assessed by flow cytometry after 3 days. Cytokines were assessed in cell-free supernatants from cultures of EV-MDSCs and from melanoma patients' CD14 ${ }^{+}$cells purified from PBMCs using a Cytokine Bead Array (CBA) (BD Biosciences), according to the manufacturer's instructions.

RNA extraction and $q P C R$ analysis. RNA was extracted from monocytes, EV-MDSCs, melanoma cells, and melanoma specimens with the mirVana miRNA Isolation Kit (Ambion). qPCR analysis was performed according to the manufacturer's instructions using Thermo Fisher Scientific and Exiqon reagents to evaluate gene and miR expression levels, respectively. The list of assays is provided in Supplemental Table 8. The endogenous controls used for normalization were GAPDH and ACTB for genes and U6 snRNA and SNORD48 for miRs. qPCR was carried out in triplicate and run on the ABI Prism 7900HT instrument, and analysis was performed using SDS software, version 2.2.2. Relative expression was determined using the $2^{-\Delta \mathrm{Ct}}$ method and expressed in arbitrary units, and FC was calculated by the $2^{-\Delta \Delta C t}$ method. For plasma and EV samples, synthetic spike-in ath-miR-159a was added to monitor RNA isolation efficiency, and the NucleoSpin
miRNA Plasma Kit (Macherey-Nagel) was used for RNA isolation. $250 \mu \mathrm{l}$ of plasma were used. Before performing RNA extraction, EV samples were treated with RNAse A (10 $\mu \mathrm{g} / \mathrm{ml}$, Sigma-Aldrich) for 1 hour at $37^{\circ} \mathrm{C}$. miR expression levels were evaluated using Thermo Fisher reagents; a preamplification step was included in this process. To evaluate MDSC-miRs in the plasma of patients, RNA was reverse transcribed and preamplified with Megaplex RT and PreAmp Primers Human Pool A and tested in duplicate with TaqMan Array MicroRNA Cards (Thermo Fisher Scientific). To set up miRNA analysis by customized cards, the system conditions relative to spike-in use, retrotranscription, and preamplification conditions were defined in titration experiments and ath-miR-159a to control RNA extraction and U6 snRNA were used for quality control. All samples were tested in the same batch of cards. The cards were run on QuantStudio 7 Flex, and analysis was performed with QuantStudio 6 and 7 Flex software.

Gene expression and miR microarray profiling. Gene expression profiles were generated using the HumanHT-12 v4 WGDASL BeadChip array (Illumina), and miR profiles were obtained using custom SurePrint Human miRNA Microarrays (Agilent). Raw gene expression data were $\log _{2}$ transformed and normalized with robust spline normalization using the lumi package (73). Probes with a detection $P$ value of less than 0.01 in at least 1 sample were kept, and for each gene, the probe with the highest detection rate was selected. Raw miR data were background corrected with the normexp method and quantile normalized using the limma package (74). Only probes with intensity higher than $10 \%$ of the 95 th percentile of the negative controls in at least 1 sample were kept. The intensities of replicate probes were averaged, and the probes with the highest mean expression across samples were selected for each miR. Differential expression analysis was carried out with the limma package. $P$ values were corrected for multiple testing using the Benjamini-Hochberg FDR. Genes and miRs with FDR of less than 0.05 and absolute FC of 1.2 or more were considered significant. All original microarray data were deposited in the NCBI's Gene Expression Omnibus database (GSE102011). Functional analyses were generated through the use of IPA (QIAGEN Inc., https://www. qiagenbioinformatics.com/products/ingenuity-pathway-analysis/) and Gene Set Enrichment Analysis (GSEA) (75). In silico evaluation of subpopulations was assessed with CIBERSORT (34).

IHC and ISH. Melanoma sections were stained for CD163 after antigen retrieval performed by heating in a pressure cooker with citrate buffer at pH 6, $5 \mathrm{mM}$ for 15 minutes and using the Dako REAL Detection System Alkaline Phosphatase/RED kit and red AP as a chromogen (Dako Agilent). Sections were scanned using the Aperio ScanScope XT systems (Aperio Technologies, Leica Microsystems), and signal quantifications were conducted by image analysis by MIAQuant as described previously (76). ISH analysis for miR visualization was performed by hybridization with double-DIG-LNA probes and positive and negative controls (Exiqon), as previously described (77). High-resolution Z stacks were acquired using Olympus BX63 equipped with a DP89 camera and cellSens software (Olympus).

Statistics. Data were analyzed using GraphPad Prism 5 and 7 software (GraphPad Software Inc.). Unless otherwise indicated, the results are presented as mean $\pm \mathrm{SD}$ and in box and whiskers plots; the box boundaries represent the 25th and 75th percentiles, the middle line is the median value, and the whiskers represent the minimum and maximum values. The results were analyzed using 2-tailed unpaired or paired Student's $t$ tests, Mann-Whitney $U$ tests, and Pearson's and 
Spearman's correlations, as specified. A $P$ value of less than 0.05 was considered significant. Multivariate analysis, simple correlations, partial correlations, and canonical correlations of variables were calculated using SAS and R statistical software. Variables were also ranked in order to standardize values before calculating the abovementioned correlations. Plasma miRs were investigated as putative prognostic factors, and their association with OS was first examined with univariable Cox's proportional hazard models. Their joint association with OS was then investigated by means of a machine-learning algorithm known as AIM. This approach was used to obtain an index score based on the following steps: (a) forward selection of the miRs significantly associated with the clinical endpoints of interest, OS and PFS, in a proportional-hazard Cox's regression model; (b) definition of an optimal cutoff for each selected miR (based on $\mathrm{Ct}$ ); and (c) construction of the index score, as an enumeration of miRs above the cutoff value. The index score built on data included 5 miRs; hence, we could split the patient cohort into 2 groups based on index score of $0-1$ (0 or $1 \mathrm{miR}$ with altered expression) or index score of 2-5 (2 or more miRs with altered expression). OS and PFS curves according to the levels of the selected score were finally estimated with the Kaplan-Meier method.

Study approval. The study protocol (code INT39/11 and INT40/11) for human studies was approved by the IRB of Fondazione IRCCS Istituto Nazionale dei Tumori and the Independent Ethics Committee. All participants, including melanoma patients and blood donors, gave informed written consent. All animal studies were approved by Regional Board of the state Baden-Wuertemberg (Karlsruhe).

\section{Author contributions}

$\mathrm{VH}, \mathrm{VV}, \mathrm{MR}, \mathrm{LR}, \mathrm{VU}$, and PA conceived the study and wrote the manuscript. VF, XH, AC, MD, ES, RS, EV, PF, ADL, BV, AG, AV,
MC, and VB performed the experiments and data analyses. EC developed image quantification software. LDG, FDB, RP, and FA conceived the experimental design in a clinical setting. LL and LM performed statistical analysis.

\section{Acknowledgments}

We thank Loris De Cecco and Marialuisa Sensi of the Platform of Integrated Biology for the genomic studies. We acknowledge Mario Santinami and Aldo Bono for their collaboration in patient accrual. We acknowledge Barbara Valeri and Annunziata Gloghini for their pathology expertise. We thank Pamela Della Mina for the TEM experiments; Paola Squarcina, Paola Deho, Simona Frigerio, and Martina Filugelli for their excellent technical assistance; and Sara Rigoletto for her experimental contributions. We thank Sascha Keller and Marcel Ramacher for their help with the mouse experiments. This work was supported by the Associazione Italiana per la Ricerca sul Cancro (AIRC) Special Program Innovative Tools for Cancer Risk Assessment and early Diagnosis 5X1000 (no. 12162 to LR), by AIRC grants IG10727 (to LR) and IG17462 (to MR), by the Cariplo Foundation (2015-0911 to VV), by awards from the Harry J. Lloyd Charitable Trust for human studies (to MR and LR), and by the European Union's Horizon 2020 Research and Innovation Programme grant agreement no. 686089 (PRECIOUS). The study was also supported in part by grants from German Research Council RTG2099 (to VU) and by the COST Action BM1404 Mye-EUNITER.

Address correspondence to: Licia Rivoltini, Unit of Immunotherapy of Human Tumors, Fondazione IRCCS Istituto Nazionale dei Tumori di Milano, Via Venezian 1, 20133 Milan, Italy. Phone 39.02.2390.3245; Email: licia.rivoltini@istitutotumori.mi.it.
1. Topalian SL, Drake CG, Pardoll DM. Immune checkpoint blockade: a common denominator approach to cancer therapy. Cancer Cell. 2015;27(4):450-461.

2. Sharma P, Hu-Lieskovan S, Wargo JA, Ribas A. Primary, adaptive, and acquired resistance to cancer immunotherapy. Cell. 2017;168(4):707-723.

3. Yarchoan M, Hopkins A, Jaffee EM. Tumor mutational burden and response rate to PD- 1 inhibition. N Engl J Med. 2017;377(25):2500-2501.

4. Chen DS, Mellman I. Elements of cancer immunity and the cancer-immune set point. Nature. 2017;541(7637):321-330.

5. Hugo W, et al. Genomic and transcriptomic features of response to anti-PD-1 therapy in metastatic melanoma. Cell. 2016;165(1):35-44.

6. Gabrilovich DI. Myeloid-derived suppressor cells. Cancer Immunol Res. 2017;5(1):3-8.

7. Ouzounova M, et al. Monocytic and granulocytic myeloid derived suppressor cells differentially regulate spatiotemporal tumour plasticity during metastatic cascade. Nat Commun. 2017;8:14979.

8. Marvel D, Gabrilovich DI. Myeloid-derived suppressor cells in the tumor microenvironment: expect the unexpected. J Clin Invest. 2015;125(9):3356-3364.

9. Bronte $\mathrm{V}$, et al. Recommendations for myeloid-derived suppressor cell nomenclature and characterization standards. Nat Commun. 2016;7:12150
10. Umansky V, Blattner C, Gebhardt C, Utikal J. The role of myeloid-derived suppressor cells (MDSC) in cancer progression. Vaccines (Basel) 2016;4(4):E36.

11. Filipazzi $P$, et al. Identification of a new subset of myeloid suppressor cells in peripheral blood of melanoma patients with modulation by a granulocyte-macrophage colony-stimulation factor-based antitumor vaccine. J Clin Oncol. 2007;25(18):2546-2553.

12. Solito S, Marigo I, Pinton L, Damuzzo V, Mandruzzato S, Bronte V. Myeloid-derived suppressor cell heterogeneity in human cancers. Ann NY Acad Sci. 2014;1319:47-65.

13. Walter S, et al. Multipeptide immune response to cancer vaccine IMA901 after single-dose cyclophosphamide associates with longer patient survival. Nat Med. 2012;18(8):1254-1261.

14. Gebhardt C, et al. Myeloid cells and related chronic inflammatory factors as novel predictive markers in melanoma treatment with ipilimumab. Clin Cancer Res. 2015;21(24):5453-5459.

15. Weber J, et al. Phase I/II study of metastatic melanoma patients treated with nivolumab who had progressed after ipilimumab. Cancer Immunol Res. 2016;4(4):345-353.

16. Valpione S, et al. Personalised medicine: Development and external validation of a prognostic model for metastatic melanoma patients treated with ipilimumab. Eur J Cancer.
2015;51(14):2086-2094.

17. Ferrucci PF, et al. Baseline neutrophils and derived neutrophil-to-lymphocyte ratio: prognostic relevance in metastatic melanoma patients receiving ipilimumab. Ann Oncol. 2016;27(4):732-738.

18. Peinado $\mathrm{H}$, et al. Melanoma exosomes educate bone marrow progenitor cells toward a pro-metastatic phenotype through MET. Nat Med. 2012;18(6):883-891.

19. Raposo G, Stoorvogel W. Extracellular vesicles: exosomes, microvesicles, and friends. JCell Biol. 2013;200(4):373-383.

20. Yáñez-Mó M, et al. Biological properties of extracellular vesicles and their physiological functions. J Extracell Vesicles. 2015;4:27066.

21. Robbins PD, Morelli AE. Regulation of immune responses by extracellular vesicles. Nat Rev Immunol. 2014;14(3):195-208.

22. Whiteside TL. Exosomes and tumormediated immune suppression. J Clin Invest. 2016;126(4):1216-1223.

23. Webber J, Yeung V, Clayton A. Extracellular vesicles as modulators of the cancer microenvironment. Semin Cell Dev Biol. 2015;40:27-34.

24. Poschke I, Mougiakakos D, Hansson J, Masucci GV, Kiessling R. Immature immunosuppressive CD14+HLA-DR-/low cells in melanoma patients are Stat3hi and overexpress CD80, CD83, and DC-sign. Cancer Res. 2010;70(11):4335-4345.

25. Gabrilovich DI, Ostrand-Rosenberg S, Bronte 
V. Coordinated regulation of myeloid cells by tumours. Nat Rev Immunol. 2012;12(4):253-268.

26. Zhao F, Falk C, Osen W, Kato M, Schadendorf D, Umansky V. Activation of p38 mitogen-activated protein kinase drives dendritic cells to become tolerogenic in ret transgenic mice spontaneously developing melanoma. Clin Cancer Res. 2009;15(13):4382-4390.

27. Danesh A, et al. Exosomes from red blood cell units bind to monocytes and induce proinflammatory cytokines, boosting T-cell responses in vitro. Blood. 2014;123(5):687-696.

28. Tomiyama T, et al. The modulation of co-stimulatory molecules by circulating exosomes in primary biliary cirrhosis. Cell Mol Immunol. 2017;14(3):276-284.

29. Condamine T, Mastio J, Gabrilovich DI. Transcriptional regulation of myeloid-derived suppressor cells. J Leukoc Biol. 2015;98(6):913-922.

30. Chow A, et al. Macrophage immunomodulation by breast cancer-derived exosomes requires Tolllike receptor 2-mediated activation of NF-кB. Sci Rep. 2014;4:5750.

31. Lam K, et al. Hmga2 is a direct target gene of RUNX1 and regulates expansion of myeloid progenitors in mice. Blood. 2014;124(14):2203-2212.

32. Zhao M, Kong L, Liu Y, Qu H. dbEMT: an epithelial-mesenchymal transition associated gene resource. Sci Rep. 2015;5:11459.

33. Schramm HM. Should EMT of cancer cells be understood as epithelial-myeloid transition? J Cancer. 2014;5(2):125-132.

34. Newman AM, et al. Robust enumeration of cell subsets from tissue expression profiles. Nat Methods. 2015;12(5):453-457.

35. Gabrusiewicz K, et al. Glioblastoma-infiltrated innate immune cells resemble MO macrophage phenotype. JCI Insight. 2016;1(2):e85841.

36. Angelova $\mathrm{M}$, et al. Characterization of the immunophenotypes and antigenomes of colorectal cancers reveals distinct tumor escape mechanisms and novel targets for immunotherapy. Genome Biol. 2015;16:64.

37. Hamm A, et al. Tumour-educated circulating monocytes are powerful candidate biomarkers for diagnosis and disease follow-up of colorectal cancer. Gut. 2016;65(6):990-1000.

38. Cook J, Zollinger R, Hagemann T. Query DataSets for GSE60601. National Center for Biotechnology Information. https://www.ncbi.nlm.nih. gov/geo/query/acc.cgi?acc $=$ gse 60601 . Updated August 09, 2018. Accessed September 21, 2018.

39. Bergenfelz C, et al. Systemic monocytic-MDSCs are generated from monocytes and correlate with disease progression in breast cancer patients. PLoS One. 2015;10(5):e0127028.

40. O'Connell RM, Zhao JL, Rao DS. MicroRNA function in myeloid biology. Blood. 2011;118(11):2960-2969.

41. Squadrito ML, Etzrodt M, De Palma M, Pittet MJ. MicroRNA-mediated control of macrophages and its implications for cancer. Trends Immunol. 2013;34(7):350-359.

42. Lee HM, Kim TS, Jo EK. MiR-146 and miR-125 in the regulation of innate immunity and inflammation. BMB Rep. 2016;49(6):311-318.

43. Challagundla KB, et al. Exosome-mediated transfer of microRNAs within the tumor microenviron- ment and neuroblastoma resistance to chemotherapy. J Natl Cancer Inst. 2015;107(7):djv135.

44. Valadi H, Ekström K, Bossios A, Sjöstrand M, Lee JJ, Lötvall JO. Exosome-mediated transfer of mRNAs and microRNAs is a novel mechanism of genetic exchange between cells. Nat Cell Biol. 2007;9(6):654-659.

45. Mittelbrunn M, et al. Unidirectional transfer of microRNA-loaded exosomes from $\mathrm{T}$ cells to antigen-presenting cells. Nat Commun. 2011;2:282.

46. Crowley E, Di Nicolantonio F, Loupakis F, Bardelli A. Liquid biopsy: monitoring cancer-genetics in the blood. Nat Rev Clin Oncol. 2013;10(8):472-484.

47. Khagi Y, et al. Hypermutated circulating tumor DNA: correlation with response to checkpoint inhibitor-based immunotherapy. Clin Cancer Res. 2017;23(19):5729-5736.

48. Schumacher TN, Scheper W. A liquid biopsy for cancer immunotherapy. Nat Med. 2016;22(4):340-341.

49. Kaneda MM, et al. PI3K $\gamma$ is a molecular switch that controls immune suppression. Nature. 2016;539(7629):437-442.

50. Zhang Y, et al. Myeloid cells are required for PD-1/PD-L1 checkpoint activation and the establishment of an immunosuppressive environment in pancreatic cancer. Gut. 2017;66(1):124-136.

51. Zhang C, Wang S, Liu Y, Yang C. Epigenetics in myeloid derived suppressor cells: a sheathed sword towards cancer. Oncotarget. 2016;7(35):57452-57463.

52. Huang-Doran I, Zhang CY, Vidal-Puig A. Extracellular vesicles: novel mediators of cell communication in metabolic disease. Trends Endocrinol Metab. 2017;28(1):3-18.

53. Mao Y, et al. Melanoma-educated CD14+ cells acquire a myeloid-derived suppressor cell phenotype through COX-2-dependent mechanisms. Cancer Res. 2013;73(13):3877-3887.

54. Hoogeveen RM, et al. Monocyte and haematopoietic progenitor reprogramming as common mechanism underlying chronic inflammatory and cardiovascular diseases. Eur Heart J. 2018;39(38):3521-3527.

55. Tcyganov E, Mastio J, Chen E, Gabrilovich DI. Plasticity of myeloid-derived suppressor cells in cancer. Curr Opin Immunol. 2018;51:76-82.

56. Millrud CR, Bergenfelz C, Leandersson K. On the origin of myeloid-derived suppressor cells. Oncotarget. 2017;8(2):3649-3665.

57. Cobos Jiménez V, Bradley EJ, Willemsen AM, van Kampen AH, Baas F, Kootstra NA. Next-generation sequencing of microRNAs uncovers expression signatures in polarized macrophages. Physiol Genomics. 2014;46(3):91-103.

58. Eigsti RL, Sudan B, Wilson ME, Graff JW. Regulation of activation-associated microRNA accumulation rates during monocyte-to-macrophage differentiation. J Biol Chem. 2014;289(41):28433-28447.

59. Essandoh K, Li Y, Huo J, Fan GC. miRNA-mediated macrophage polarization and its potential role in the regulation of inflammatory response. Shock. 2016;46(2):122-131.

60. Taganov KD, Boldin MP, Chang KJ, Baltimore D. NF-kappaB-dependent induction of microRNA miR-146, an inhibitor targeted to signaling proteins of innate immune responses. Proc Natl Acad Sci US A. 2006;103(33):12481-12486.

61. Dudda JC, et al. MicroRNA-155 is required for effector CD8+ T cell responses to virus infection and cancer. Immunity. 2013;38(4):742-753.

62. Li L, et al. MicroRNA-155 and MicroRNA-21 promote the expansion of functional myeloid-derived suppressor cells. JImmunol. 2014;192(3):1034-1043.

63. Tili E, Croce CM, Michaille JJ. miR-155: on the crosstalk between inflammation and cancer. Int Rev Immunol. 2009;28(5):264-284.

64. Elton TS, Selemon H, Elton SM, Parinandi NL. Regulation of the MIR155 host gene in physiological and pathological processes. Gene. 2013;532(1):1-12.

65. Huang J, et al. MiR-155 is upregulated in patients with active tuberculosis and inhibits apoptosis of monocytes by targeting FOXO3. Mol Med Rep. 2015;12(5):7102-7108.

66. Chen S, et al. Host miR155 promotes tumor growth through a myeloid-derived suppressor cell-dependent mechanism. Cancer Res. 2015;75(3):519-531.

67. Chaudhuri AA, et al. MicroRNA-125b potentiates macrophage activation. JImmunol. 2011;187(10):5062-5068.

68. Shyh-Chang N, Daley GQ. Lin28: primal regulator of growth and metabolism in stem cells. Cell Stem Cell. 2013;12(4):395-406.

69. UCSC Genome Browser on Human Dec. 2013 GRCh38/hg38 Assembly. University of California Santa Cruz Genomics Institute. https://genome. ucsc.edu/cgi-bin/hgTracks?db=hg38\&lastVirt ModeType $=$ default\&lastVirtModeExtraState $=$ \&virtModeType $=$ default\&virtMode $=0$ \&non VirtPosition=\&position=chr11\%3A121300001\% 2D124000000\&hgsid=695238597 Xy4ztE2 Wm6IfOlPFMDQAR8iF9DFh.

Accessed October 29, 2018

70. Hildebrand D, et al. Hsa-miR-99b/let-7e/miR125 a cluster regulates pathogen recognition receptor-stimulated suppressive antigen-presenting cells. Front Immunol. 2018;9:1224.

71. Daniotti M, et al. BRAF alterations are associated with complex mutational profiles in malignant melanoma. Oncogene. 2004;23(35):5968-5977.

72. Théry C, Amigorena S, Raposo G, Clayton A. Isolation and characterization of exosomes from cell culture supernatants and biological fluids. Curr Protoc Cell Biol. 2006; Chapter 3:Unit 3.22.

73. Du P, Kibbe WA, Lin SM. lumi: a pipeline for processing Illumina microarray. Bioinformatics. 2008;24(13):1547-1548.

74. Ritchie ME, et al. limma powers differential expression analyses for RNA-sequencing and microarray studies. Nucleic Acids Res. 2015;43(7):e47.

75. Subramanian A, et al. Gene set enrichment analysis: a knowledge-based approach for interpreting genome-wide expression profiles. Proc Natl Acad Sci US A. 2005;102(43):15545-15550.

76. Casiraghi E, et al. MIAQuant, a novel system for automatic segmentation, measurement, and localization comparison of different biomarkers from serialized histological slices. Eur J Histochem. 2017;61(4):2838.

77. Gualeni AV, Volpi CC, Carbone A, Gloghini A. A novel semi-automated in situ hybridisation protocol for microRNA detection in paraffin embedded tissue sections. JClin Pathol. 2015;68(8):661-664. 